\title{
Reflexiones sobre la experiencia en el programa de residencia en Medicina Familiar y Comunitaria en España
}

\author{
Reflexões sobre a experiência no programa de residência em Medicina de Família e Comunidade na Espanha \\ Reflections on the experience in the Family and Community Medicine residency programme in Spain
}

Lourdes Luzón Oliver. Secretaria Municipal de Saúde do Rio de Janeiro (SMSRJ). Rio de Janeiro, RJ, Brasil. lourdesluzon@gmail.com (Autora correspondiente)

Verónica Casado Vicente. Centro de Salud Docente y Universitario Parquesol. Valladolid, Castilla y León, España. vcasadovicente@gmail.com

\section{Resumen}

En este artículo se relata la experiencia de una médica de familia a su paso por la Universidad, por el examen Médico Interno Residente (MIR) y por el programa de residencia en Medicina Familiar y Comunitaria, así como expectativas laborales, y su visión de futuro de la Atención Primaria.

\section{Resumo}

Este artigo relata a experiência de uma médica de família em sua trajetória pela universidade, o exame Médico Interno Residente (MIR) e sua vivência no programa de residência em Medicina de Família e Comunidade, assim como as expectativas laborais e sua visão de futuro da Atenção Primaria.

\section{Abstract}

This article describes the experience of a family physician in her university trajectory, the Physician Internship Resident exam (MIR), and her experience in the Family and Community Medicine residence programme as well as the career prospects and her vision on the future of primary care.

\section{El paso por la facultad}

Mi comienzo en la universidad, como la de todo universitario, fue un salto a lo desconocido. Con 18 años, preguntábamos a nuestros mayores su experiencia, y escuchábamos de todo, bueno, malo y regular. En mi caso fue un doble salto al vacío porque la Facultad de Medicina de Albacete en el año 1998 estaba abriendo sus puertas para sus primeros "conejillos de indias", con una metodología totalmente innovadora en Espańa, basada en el "autoaprendizaje", y en el estudio autodirigido e independiente. Todo esto fue un "shock" porque no esperábamos nada parecido, y sin duda sería un desafío para todos, decano, profesores, profesionales sanitarios, secretariado, alumnado y pacientes. Era un programa de gran envergadura basado en objetivos a cumplir y en competencias a adquirir. Lo que le diferenciaba de otras facultades era el abordaje integral de las asignaturas, el modelo de clases, desapareciendo el modelo antiguo pasivo de clases magistrales y sustituido por un modelo activo en el que los alumnos éramos los protagonistas, apoyados por el cuerpo de profesores; la elevada carga horaria práctica, el gran número de exámenes que realizábamos para reforzar conceptos, los exámenes ECOE (OSCE) desde el tercer año, y algunas otras novedades como el tener la asignatura de Atención Primaria durante el segundo año de carrera. Todo ello hacía de él un programa diferenciado a lo que había hasta ese momento en España.
Palabras clave:

Medicina Familiar y Comunitaria Internado y Residencia Educación Médica

Palavras-chave: Medicina de Família e Comunidade Internato e Residência Educação Médica

Keywords: Family Practice Internship and Residency Education, Medical 
Claro que ese no fue mi primer contacto con la Atención Primaria, ya lo había tenido mucho tiempo atrás como usuaria ya que, en Espańa, todos los pacientes, ricos y pobres, feos y guapos tenemos un médico de familia asignado desde el nacimiento hasta la muerte. Creo que es una gran ventaja contar con la asignatura desde el comienzo de la carrera, ya que antes de tener un conocimiento más focal de las diferentes especialidades pudimos percibir la dimensión de la AP para un país, aprendimos como está estructurado nuestro sistema de AP, comenzamos a discutir el concepto de salud de la OMS, implicación de determinantes sociales, empezamos a escuchar hablar de los atributos de la AP, de la longitudinalidad del cuidado. Cuando decidí estudiar medicina no me imaginaba siendo otra cosa que Médico de Familia, por la dimensión sociosanitaria de la especialidad, entre otras cosas. A lo largo de la carrera me fui apasionando por cada una de las especialidades, y cuando terminé percibí que me gustaba todo, y me reafirmé en mi idea inicial de no querer ser especialista de órganos sino de ser especialista en personas, saber de todo, ver al paciente desde otra perspectiva, quería atender enfermos no enfermedades.

\section{El paso por el MIR (Médico Interno Residente)}

Después de la facultad viene el examen "MIR", que provocaba pánico en todo estudiante de medicina, sobre todo cuando iba llegando al final. Aquel momento recuerdo que era vivido como una especie de histeria colectiva. La presión era alta por parte de las universidades, que siempre quieren que sus alumnos obtengan los mejores puestos en la clasificación, de los familiares y por supuesto del propio alumno. Aunque tenía claro que quería escoger MF y que para eso no iba a tener dificultades por el número de plazas convocadas, que siempre es mucho mayor que del resto, los nervios estaban a flor de piel. Fueron 8 meses de estudio muy intensos para intentar demostrar lo mejor de nosotros mismos, con una alta competitividad. Pero mi duda siempre era ¿̇será que en esa prueba seremos capaces de demostrar no solo nuestros conocimientos como también potencialmente nuestras habilidades y cualidades como futuros profesionales? Ciertamente no. Es una prueba por un lado justa porque somete a todos los aspirantes al mismo tipo de prueba y con las mismas condiciones, pero por otro lado otorga demasiado peso a una única prueba, agotadora, de 5 horas de duración, y poco al trabajo desarrollado a lo largo de 6 años.

Obtenidos los resultados llegó el momento de escoger unidad docente (UD), ¿Cual sería mejor? ¿Qué ventajas me podrían ofrecer unas sobre otras? Comenzaba la búsqueda de informaciones de colegas y llamadas a diferentes UUDD del país para obtener referencias. Finalmente me decidí por una que parecía tener bastantes años de experiencia, y me ofrecía una amplia gama de rotatorios fijos y optativos tanto internos como externos, que me conquistó. Mi principal duda era, entre otras, escoger una UD vinculada a un Hospital Terciario (que implicaba pacientes más complejos y posibilidad de rotatorios diferenciados) o un hospital menor (donde no existen residentes de otras especialidades y el residente de MFC es el protagonista de todo). Al final me decidí por la primera opción pero más por las características de la UD en sí, y porque además estaba localizada en una ciudad como Zaragoza.

\section{El paso por la residencia}

Fueron cuatro ańos muy intensos a nivel profesional y personal. Pasamos de estudiantes a ser profesionales en formación contratados, asumiendo una independencia financiera y progresivamente una autonomía también profesional. Normalmente las rotaciones eran de un mes (otorrinolaringología, ginecología, dermatología, cardiología...), aunque teníamos otras más diferenciadas como medicina interna o psiquiatría en las que estábamos 3 meses o radiología 2 meses.

Rotaciones, cursos, guardias infernales de 24 horas en urgencias, pediatría, traumatología, inicio en la participación de congresos, formación de estrechos vínculos personales y profesionales. A veces sobrevenía la sensación de estar prestando servicio a un menor coste, pero es cierto que la sensación asfixiante de un nudo en el estómago de las primeras guardias se iba transformando en experiencia y adquisición de habilidades. Pudimos conocer las interconexiones entre AP y niveles secundarios, a veces nos encontrábamos con "nuestros pacientes" cuando estábamos rotando en otros servicios, lo que agregaba la importancia de la coordinación del cuidado. Dio para conocer lo mejor y lo "mejorable" del sistema.

También hubo rotaciones "especiales" como Cuidados Paliativos, "Proyecto Hombre" y mi estancia en Brasil dos meses. En estas rotaciones no sólo aprendí a tratar enfermos de forma diferenciada sino que aprendí unos valores que difícilmente se encuentran en los libros. Y sin duda lo más enriquecedor fue mi paso por el centro de salud. Fui condicionada a cambiar de tutora durante el primer año, en el que había pasado mis primeros meses en el centro de salud. Esa indefinición temporal 
hizo que durante unos meses estuviese más desvinculada del centro de salud, lo que me hizo perder algunas oportunidades de vínculo con la unidad. Finalmente cuando volví al centro me sentí como en casa. Mi tutora trabajaba en ese equipo desde hacía más de 10 años y tenía un gran conocimiento de sus pacientes. Fue fácil trabajar junto a ella y aprender de ella y con ella y con el resto de los profesionales que siempre ponían en común sus fortalezas y sus debilidades. El equipo de profesionales era fantástico, con ellos aprendí mucho más que las competencias descritas por el programa, aprendí a incluir la perspectiva de género en mi abordaje habitual y así como el manejo de competencias culturales o el análisis de casos enfocados en la seguridad del paciente, por ejemplo. Era un centro que escogí también por sus características poblaciones de exclusión social e inmigración, con una gran demanda asistencial en el que podíamos llegar a atender hasta 40-50 pacientes por día sin dejar de hacer avisos domiciliares acompañando a nuestros pacientes hasta el fin, intentando ofrecer una muerte digna. Y no dejábamos de aportar el lado docente a nuestro trabajo cotidiano, a veces no tanto en las prisas de la consulta, pero sí llevándolo al programa prácticamente diario de sesiones clínicas, algunas más focales y sólo de médicos, y otras (una vez por semana) haciendo participes a todos los profesionales de la clínica.

Casi pasaron cinco ańos del término de mi especialidad y con el paso del tiempo tengo más claridad sobre la fortaleza y la potencialidad del programa. Infelizmente el nuevo programa se estaba implantando cuando yo inicié la residencia, por lo que hubo propuestas de las que, con mucha envidia hoy, no fui participe. Sin embargo dada la larga trayectoria de la especialidad en España, y de la UD que escogí puedo decir que participé de un programa sólido, claro y fuerte en el que se apostaba por formar médicos polivantes.

Creo que es un programa que tiene mucho que aportar tanto por su estructura como por su contenido y que puede ser adaptado para programas de otros países con otras características. Tiene gran valor la diferenciación de unas competencias esenciales y otras transversales que se complementan, así como la definición de unos niveles de prioridad para que todos los residentes cumplan unos mínimos de formación comunes.

\section{¿Y ahora qué?}

Con el fin de la residencia viene la incertidumbre de la búsqueda de trabajo. Somos de los profesionales que más tarde iniciamos esta peregrinación tras 6 ańos de carrera, casi uno de preparación para el MIR, con suerte, y 4 o 5 años de especialidad. En ese momento la mayoría ya comienzan a tener sus vidas medio definidas, y ahí es cuando viene el problema.

Terminé la residencia con la crisis ya instaurada en España en 2009, con todo lo que se avecinaba para el SNS, y como siempre la más perjudicada sería la Atención Primaria. En Aragón donde me formé, se ofrecían contratos por días o por horas, este era el panorama. En otras regiones en ese momento la situación estaba algo mejor pero la realidad es que tristemente lo que se ofrece actualmente son contratos inestables, sujetos a la movilidad del profesional y la dificultad de establecer vínculo profesional y personal en una región determinada. Infelizmente nuestros gestores y políticos están en un momento en el que han borrado de sus mentes privilegiadas la eficiencia de un sistema con una AP estructurada y fuerte.

Creo firmemente que nuestra especialidad es una fuente de oportunidades para los locos que nos atrevemos a escogerla y para la sostenibilidad del SNS. Y a pesar del reconocimiento por colegas de profesión y por la propia sociedad, las instituciones competentes continúan bloqueando el ejercicio de competencias que serían más coste/efectivas si las realizasen los MF que los especialistas focales. Por tanto tenemos la obligación, no solo profesional sino también moral de continuar haciendo visible nuestro trabajo para colocar la especialidad en el lugar que se merece.

Mi "visión", es decir hacia dónde a mi me gustaría que fueran las cosas en mi país y todos los países es que a corto plazo se haya valorizado la Atención Primaria como función central de los sistemas sanitarios y, la Medicina Familiar y Comunitaria como disciplina, especialidad y profesión sanitaria, que su valor intrínseco y extrínseco coincidan porque las autoridades competentes lo han convertido en un objetivo claro y fundamental. Que finalmente se entienda que un sistema sanitario que no defiende la eficacia de nivel está abocado a la ineficiencia y a la inequidad, que un sistema sanitario viable es el que equilibra fuerzas y construye un sistema realmente integrado y coordinado desde la base, entre los profesionales, favoreciendo la coalición de especialistas con el objetivo final de mejorar la salud. Que se entienda que la Medicina Familiar y Comunitaria debe tener un papel nuclear en la formación de grado, en la troncalidad y en la formación especializada y que en un escenario de crisis, con un entorno de envejecimiento, cronicidad de procesos, alta demanda asistencial, costes crecientes, necesaria ambulatorización de los cuidados, los decisores políticos opten por hacer "gestión basada en la evidencia", eligiendo las estrategias más pertinentes y adoptando las medidas más coste/efectivas, por lo que financian adecuadamente 
la AP, la trasladan poder para organizarse y colocan la atención primaria no sólo como la puerta de entrada de los sistemas sanitarios sino como función central del sistema. Y consideran, como no puede ser de otra manera, que una estrategia clave es apostar de forma clara por la formación en Medicina Familiar y Comunitaria tanto en el grado como en formación especializada, porque como decía Barbara Starfield "la MFyC debe dar forma a la reforma y no al revés".

Nota da RBMFC: este artigo complementa e expande a compreensão sobre a formaçáo em medicina de família e comunidade na Espanha, tema discutido mais detalhadamente no artigo "Razones, retos y aportaciones del programa de la especialidad de medicina familiar y comunitaria en España". Desse modo, os leitores devem consultar o artigo mencionado para acessar a listagem de referências disponibilizada pelas autoras. 